\title{
Deubiquitinase USP48 promotes ATRA-induced granulocytic differentiation of acute promyelocytic leukemia cells
}

\author{
LIANLIAN LI ${ }^{1}$, YONG WANG ${ }^{2}$, XIAOYU ZHANG ${ }^{1}$, GUANHUA SONG ${ }^{1}$, QIANG GUO ${ }^{1}$, ZHIYONG ZHANG $^{1}$, \\ YUTAO DIAO $^{1}$, HAIPENG YIN ${ }^{1}$, HONGYAN LIU ${ }^{1}$ and GUOSHENG JIANG ${ }^{1}$ \\ ${ }^{1}$ Institute of Basic Medicine, Shandong Academy of Medical Sciences, Jinan, Shandong 250062; \\ ${ }^{2}$ Shandong Xinchuang Biotechnology Co., Ltd., Jinan, Shandong 250102, P.R. China
}

Received February 2, 2018; Accepted May 4, 2018

DOI: $10.3892 /$ ijo.2018.4440

\begin{abstract}
All-trans retinoic acid (ATRA) has been used for the treatment of acute promyelocytic leukemia (APL). However, its molecular mechanisms of action are unclear. Ubiquitinspecific protease 48 (USP48) is a deubiquitinase enzyme that can post-translationally remove ubiquitin molecules from substrates. In the present study, the role of USP48 in ATRA-induced differentiation of APL cells was studied. The expression of USP48 decreased following ATRA treatment. Functionally, overexpression of USP48 using electroporationmediated delivery inhibited the proliferation of APL cells and promoted ATRA-mediated differentiation. The inverse observations were made upon siRNA-mediated knockdown of USP48. Furthermore, the expression of USP48 was increased in the nucleus upon ATRA exposure for $\leq 24 \mathrm{~h}$, suggesting that USP48 was translocated into the nucleus. Interestingly, regulation of p65, a substrate of USP48, did not contribute to the downstream mechanism of ATRA-induced differentiation of APL cells. In addition, upstream mechanistic studies demonstrated that the expression of USP48 was regulated by microRNA-301a-3p. In conclusion, the present study highlights the function of USP48 in the ATRA-induced granulocytic differentiation of APL cells and provides a theoretical basis for identifying novel targets for differentiation therapy of APL.
\end{abstract}

\section{Introduction}

Acute promyelocytic leukemia (APL) is a type of acute myeloid leukemia (AML), characterized by the expression of retinoic acid receptor alpha $(R A R \alpha)$ fusion genes, including

Correspondence to: Professor Hongyan Liu or Professor Guosheng Jiang, Institute of Basic Medicine, Shandong Academy of Medical Sciences, 18877 Jingshi Road, Jinan, Shandong 250062, P.R. China E-mail: hyliudycui@aliyun.com

E-mail: jiangguosh@163.com

Key words: ubiquitin-specific protease 48, deubiquitinase, all-trans retinoic acid, differentiation, leukemia
$P M L-R A R \alpha, N P M-R A R \alpha$ and $P L Z F-R A R \alpha$, resulting in blockage of myeloid differentiation and aberrant self-renewal of promyelocytic cells $(1,2)$. Fortunately, the majority of patients with APL achieve complete remission upon treatment with all-trans retinoic acid (ATRA) via degradation of RAR $\alpha$ fusion proteins $(3,4)$. However, ATRA therapy has a number of disadvantages, including drug resistance and a high recurrence rate $(5,6)$. The key molecular mechanism for induced differentiation of leukemia cells has not been clarified. Therefore, identifying the key molecular target of differentiation disorders is vital for the diagnosis and treatment of leukemia.

The ubiquitin proteasome system (UPS), including ubiquitination and deubiquitination, is accountable for the majority of recycling and degradation of proteins within the cell. Therefore, it regulates pathological changes, including abnormalities of the immune system and tumor cells (7-11). Ubiquitination, a common form of post-translational modification (PTM), is the process by which ubiquitin attaches to lysine residues on target proteins via a 3-enzyme cascade reaction that involves the ubiquitin-activating enzyme (E1), ubiquitinconjugating enzyme (E2) and ubiquitin ligase (E3) $(12,13)$. This PTM is reversible by deubiquitination by deubiquitinating enzymes (deubiquitinases or DUBs), which can hydrolytically remove ubiquitin from protein adducts (14-20). In humans, there are $>100$ types of DUBs, which can be divided into 5 families: Ubiquitin-specific proteases (USPs), ubiquitin carboxyl-terminal hydrolases, ovarian tumor proteases, JAB1/MPN/MOV34 metalloenzymes, and Machado-Josephin domain proteases (19). USP48 is a member of the USP family, and there are 53 USP genes in the human genome (21). Previous studies have shown that DUBs participate in cellular functions, including protein quality control and degradation, DNA damage and repair, RNA transcription and processing, and signal transduction (22).

In the present study, the role of USP48 in promoting ATRAinduced differentiation of leukemia cells was examined. It was determined that ATRA-induced differentiation was not attributable to the regulation of p65, a substrate of USP48. In addition, it was indicated that the expression of USP48 was regulated by microRNA-301a-3p. These data suggest that dysregulation of USP48 may be an underlying mechanism for the abnormal differentiation of APL, implicating USP48 as a potential therapeutic target for APL. 


\section{Materials and methods}

Cell culture and ATRA treatment. The human acute promyelocytic leukemia cell lines, NB4 and HL60, and the human acute monocytic leukemia cell line, THP1, and 293T cells were purchased from the American Type Culture Collection (Manassas, VA, USA). Leukemia cells were cultured in RPMI-1640 medium and 293T cells cultured in DMEM medium supplemented with $10 \%$ fetal bovine serum (FBS; HyClone; GE Healthcare, Chicago, IL, USA), $100 \mathrm{U} / \mathrm{ml}$ penicillin and $100 \mu \mathrm{g} / \mathrm{ml}$ streptomycin, and incubated at $37^{\circ} \mathrm{C}$ in an atmosphere with $5 \% \mathrm{CO}_{2}$. Leukemia cells were treated with $1 \mu \mathrm{M}$ ATRA or phorbol-12-myristate-13-acetate (PMA; Sigma-Aldrich; Merck KGaA, Darmstadt, Germany).

Wright-Giemsa staining and microarray analysis. Leukemia cells were treated with $1 \mu \mathrm{M}$ ATRA for $72 \mathrm{~h}$. Wright-Giemsa staining was performed using a Wright-Giemsa staining kit (Beijing Solarbio Science \& Technology Co., Ltd., Beijing, China), according to the manufacturer's protocol. For microarray analysis, total RNA was extracted from HL60 cells using TRIzol (Thermo Fisher Scientific, Inc., Waltham, MA, USA), according to the manufacturer's protocol. An Affymetrix Gene Chip Human Genome U133 Plus 2.0 Array analysis was performed by Beijing CapitalBio Technology Co., Ltd. (Beijing, China).

Reverse transcription-quantitative polymerase chain reaction $(R T-q P C R)$. Total RNA was extracted from cells using TRIzol (Thermo Fisher Scientific, Inc.) according to the manufacturer's protocol. RNA was reverse-transcribed into cDNA using PrimeScript ${ }^{\mathrm{TM}}$ Reverse Transcriptase (Takara Bio, Inc., Otsu, Japan) for USP48 and a miRcute Plus miRNA First-Strand cDNA Synthesis kit (Tiangen Biotech Co., Ltd., Beijing, China), according to the manufacturer's protocol, for microRNA. The cDNAs were used as templates in RT-qPCR with the following primers: USP48, forward, 5'-TGGAGCCACTTGTTATGT-3' and reverse, 5'-GGATCAATGTATCGCCTA-3'; GAPDH, forward, 5'-ACAACTTTGGTATCGTGGAAGG-3' and reverse, 5'-GCCATCACGCCACAGTTTC-3'; the microRNA primers were purchased from Tiangen Biotech. RT-qPCR was performed using an Applied Biosystems 7500 real-time system using UltraSYBR Mixture (CWBiotech, Beijing, China) for USP48, and miRcute Plus miRNA qPCR Detection kit (cat. no. FP40101; Tiangen Biotech) for microRNA. The following conditions were used for PCR: $10 \mathrm{sec}$ at $95^{\circ} \mathrm{C}, 40$ cycles of $5 \mathrm{sec}$ at $60^{\circ} \mathrm{C}$, $10 \mathrm{sec}$ at $72^{\circ} \mathrm{C}$ and $30 \mathrm{sec}$ at $65^{\circ} \mathrm{C}$. Relative quantity of expression was calculated using the $2^{-\Delta \Delta \mathrm{Cq}}$ method (23). USP48 and microRNA expression were normalized to GAPDH and $U 6$ expression, respectively.

Western blotting. Western blot analyses were performed as previously described (24). The following primary antibodies were used: USP48 (dilution, 1:1,000; cat. no. ab72226; Abcam, Cambridge, UK), p65 (dilution, 1:1,000; cat. no. 6956; Cell Signaling Technology, Inc., Danvers, MA, USA), tubulin (dilution, 1:15,000; cat. no. RLM3030; Ruiyingbio, Suzhou, Jiangsu, China), histone H3 (dilution, 1:20,000; cat. no. GB13102-1), and GAPDH (dilution, 1:10,000; cat. no. GB13002-m-1) (both from Servicebio, Wuhan, China).
Cell proliferation assay. A Cell Counting Kit-8 (CCK-8) assay (Solarbio, Beijing, China) was used to detect cell proliferation, according to the manufacturer's instructions. In brief, $1 \times 10^{4}$ cells in $100 \mu 1$ medium were plated per well of a 96-well plate in triplicate. Following incubation for 0,24 or $48 \mathrm{~h}, 10 \mu \mathrm{l}$ CCK-8 solution was added per well, and incubated for an additional $3 \mathrm{~h}$ at $37^{\circ} \mathrm{C}$. Absorbance was measured at $450 \mathrm{~nm}$ using a microplate reader (SynergyHTX; BioTek Instruments, Inc., Winooski, VT, USA).

Flow cytometry $(F C M)$. A total of $1 \times 10^{6}$ cells treated with $1 \mu \mathrm{M}$ ATRA were washed with serum-free RPMI-1640 medium and then resuspended in serum-free RPMI-1640 medium. Cell cycle analysis and apoptosis assessment were performed using Annexin V/proprium iodide (PI) kit (cat. no. C1052; Beyotime Biotechnology, Shanghai, China) and analyzed using a flow cytometer (NovoCyteD1040; ACEA Biosciences, Inc., San Diego, CA, USA) with NovoExpress1.2.1 software. To detect cluster of differentiation (CD)11b expression, a FITC-conjugated anti-human CD11b antibody (dilution, 1:50; cat. no. 555388; BD Pharmingen; BD Biosciences, Franklin Lakes, NJ, USA) was added to the cells and incubated at $4^{\circ} \mathrm{C}$ for $30 \mathrm{~min}$. Cells were washed with PBS and analyzed using a flow cytometer (Epics XL4C) with a EXPO ${ }^{\mathrm{TM}} 32$ ADC Analysis Software (both from Beckman Coulter, Inc., Brea, CA, USA).

Immunofluorescence staining. A total of $2 \times 10^{5}$ cells were centrifuged at $400 \mathrm{xg}$ for $2 \mathrm{~min}$ at room temperature and fixed onto slides with $4 \%$ paraformaldehyde for $10 \mathrm{~min}$. Then cells were permeabilized with $0.5 \%$ Triton X-100 for $20 \mathrm{~min}$ at room temperature. After blocking with 3\% BSA for $30 \mathrm{~min}$ at room temperature, USP48 (dilution, 1:100; cat. no. ab72226; Abcam) and p65 (dilution, 1:100; cat. no. 6956; Cell Signaling Technology, Inc.) primary antibodies were incubated with the slides at $4^{\circ} \mathrm{C}$ overnight. Samples were then incubated with a goat-anti-rabbit-CY3 (dilution, 1:300; cat. no. GB21303) or goat-anti-mouse-488 nm (dilution, 1:400; cat. no. GB21301) (both from Servicebio) fluorophore-conjugated IgG secondary antibodies. A total of $100 \mu 1$ DAPI (cat. no. G1012; Servicebio) was added per slide and incubated at room temperature for $10 \mathrm{~min}$ to stain the nuclei and. The images were acquired on a Nikon Eclipse C1 fluorescence microscope (Nikon Corporation, Tokyo, Japan).

Transfection. NB4 cells were transfected using a BTX ECM 830 electroporator (BTX Harvard Apparatus, San Diego, CA, USA) with one $125 \mathrm{~V}$ pulse for $15 \mathrm{msec}$. The cells were then transferred to RPMI-1640 medium supplemented with $10 \%$ FBS and cultured for 24-48 h. For overexpression, USP48 was cloned into a pFlag-CMV-2 plasmid. Empty pFlag-CMV-2 plasmids were used as a negative control. A total of $10 \mu \mathrm{g}$ plasmid was used to transfect $5 \times 10^{6} \mathrm{NB} 4$ cells/well for $48 \mathrm{~h}$. Transient silencing was performed by transfecting the following small interfering RNAs (siRNAs) into $2 \times 10^{6} \mathrm{NB} 4$ cells/well: siUSP48-1, 5'-GCAGUUCUGUGGAGAAUAUTT AUAUUCUCCACAGAACUGCTT-3'; siUSP48-2, 5'-GCC CAACACUACUGUUCAATTUUGAACAGUAGUGUUGGG CTT-3'; siUSP48-3, 5'-GCUGGUAGAUCGGGAUAAUTTA UUAUCCCGAUCUACCAGCTT-3', and negative control, 
A

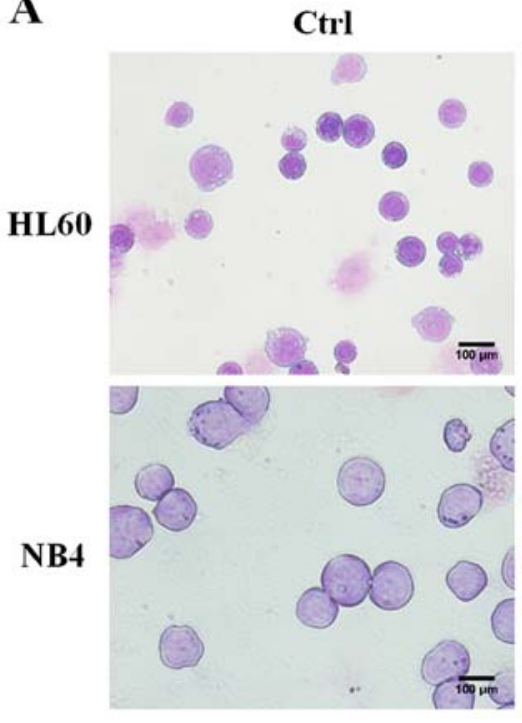

ATRA

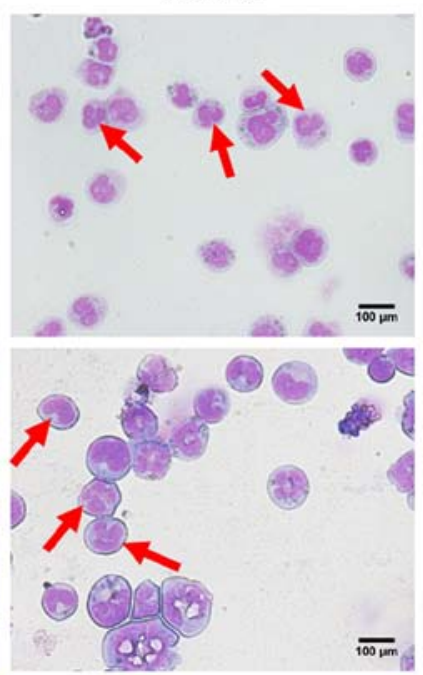

B

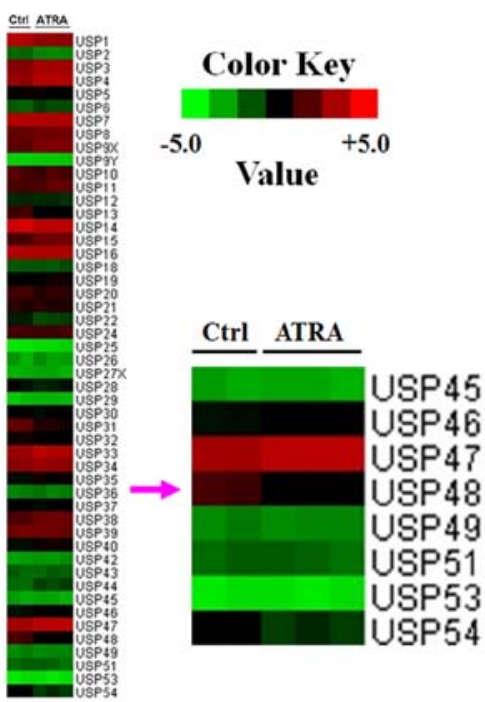

Figure 1. Differentiation of APL cells by ATRA. (A) Morphological changes of HL60 and NB4 cells following ATRA treatment. The red arrow indicates cells with lobulated nuclei. (B) A cluster heat-map from microarray demonstrates USP48 mRNA expression in HL60 cells treated with or without ATRA. APL, acute promyelocytic leukemia; ATRA, all-trans retinoic acid; USP48, ubiquitin-specific protease 48.

\section{5'-UUCUCCGAACGUGUCACGUTTACGUGACACGUUCG} GAGAATT-3'. A total of $20 \mu 1$ siRNA was transfected per well, at a stock concentration of was $1 \mathrm{OD} / 125 \mu \mathrm{l}$ RNase-free $\mathrm{H}_{2} \mathrm{O}$, for $48 \mathrm{~h}$. hsa-miR-301a-3p mimics and inhibitor (cat. nos. miR 10000688 and miR20000688, respectively) and negative control miRNAs (cat. nos. miR01101 and miR02101) (both from Guangzhou RiboBio Co., Ltd., Guangzhou, China) were transfected into $2 \times 10^{6}$ cells for $48 \mathrm{~h}$, using $20 \mu \mathrm{l}$ miRNA at a stock concentration of $5 \mathrm{nmol} / 250 \mu \mathrm{l}$ R Nase-free $\mathrm{H}_{2} \mathrm{O}$. The transfection efficiency was confirmed by detecting the mRNA or protein expression levels of USP48 using RT-qPCR or western blotting, as aforementioned.

Luciferase assay. With wild-type 3' untranslated region (UTR) (USP48-WT) or mutant 3'UTR (USP48-Mut) sequence of USP48 was cloned to a Dual-Luciferase Reporter vector (pmiR-RB-REPORT ${ }^{\mathrm{TM}}$; Guangzhou RiboBio Co., Ltd.). 293T cells were co-transfected with negative control or miR-301a-3p mimic and Dual-Luciferase Reporter plasmids carrying USP48-WT or USP48-Mut of USP48 using Lipofectamine 2000 (Thermo Fisher Scientific, Inc.). After 48 h, luciferase activities were measured with a Dual-Glo ${ }^{\circledR}$ Luciferase Assay system (Promega, Madison, WI, USA), according to the manufacturer's protocol. Renilla luciferase activity was used to normalize the Firefly luciferase activity of the reporter construct.

miRNA prediction. To investigate the regulation of USP48 expression, prediction of microRNAs targeting USP48 was performed using TargetScanHuman 7.0 (http://www.targetscan. org/vert_70/).

Survival analysis. The USP48 gene was analyzed using the cBioPortal database (http://www.cbioportal.org) $(25,26)$. All samples analyzed were derived from acute myeloid leukemia cases (The Cancer Genome Atlas; dataset, NEJM2013) (27). A Z-score threshold of \pm 2.0 was used for analysis of mRNA data (RNA Seq V2 RSEM; log; n=173).
Statistical analysis. Data are presented as the mean \pm standard deviation of $\geq 3$ experiments. Statistical analysis was performed using SPSS 13.0 software (SPSS Inc., Chicago, IL, USA) and GraphPad Prism 5.0 (GraphPad Software, Inc., La Jolla, CA, USA). Student's t-test was used to analyze the difference between 2 groups, and one-way analysis of variance was performed to analyze the difference between $\geq 3$ groups, followed by Dunnett's test. Survival was analyzed using the Kaplan-Meier model. $\mathrm{P}<0.05$ was considered to indicate a statistically significant difference.

\section{Results}

USP48 expression decreases during ATRA-induced granulocytic differentiation of APL cells. To systematically explore the underlying mechanism of abnormal granulocytic differentiation in APL, leukemia cells were treated with or without $1 \mu \mathrm{M}$ ATRA for $72 \mathrm{~h}$. Morphological analysis, the emergence of nuclear lobulation and pyknosis (Fig. 1A) confirmed the differentiation of APL cells induced by ATRA. In addition, gene microarray analysis was performed and the gene expression profiles of HL-60 cells were changed following ATRA treatment. Comparison of the expression variation of USP family members revealed that the expression of USP48 was decreased in HL60 cells treated with ATRA (Fig. 1B). RT-qPCR and western blotting revealed that ATRA stimulation significantly downregulated USP48 expression at the mRNA and protein levels in NB4 and HL-60 cells after $24 \mathrm{~h}$ treatment compared with untreated controls (Fig. 2A and B). In contrast, decreased expression of USP48 was not stimulated by PMA treatment in THP1 cells (Fig. 2C). These results indicate that USP48 expression was decreased during ATRA-induced granulocytic differentiation of APL cells.

USP48 inhibits the proliferation and promotes the ATRAinduced differentiation of NB4 cells. To investigate the role of USP48 in cell proliferation, a CCK-8 assay was performed, 
A

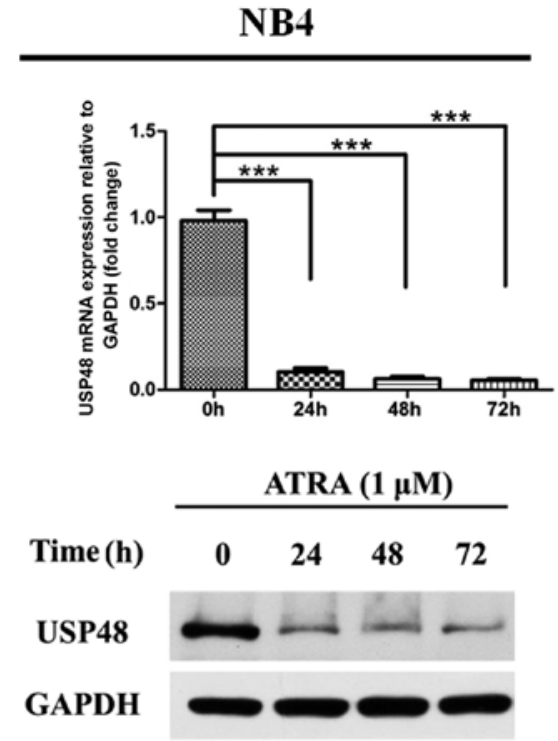

C
B

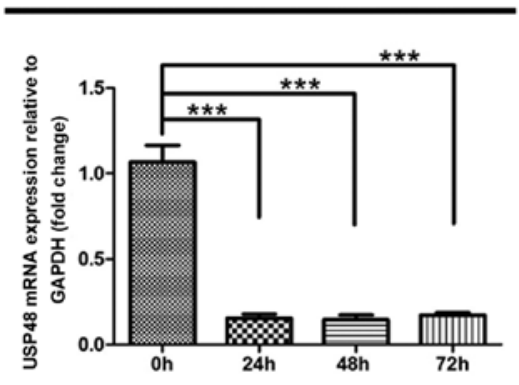

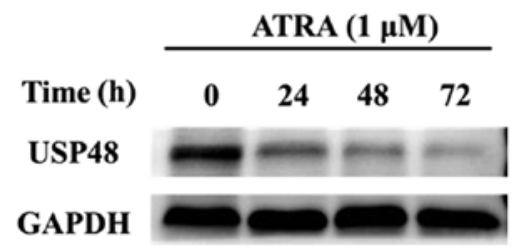

THP1

$\operatorname{PMA}(1 \mu \mathrm{M})$

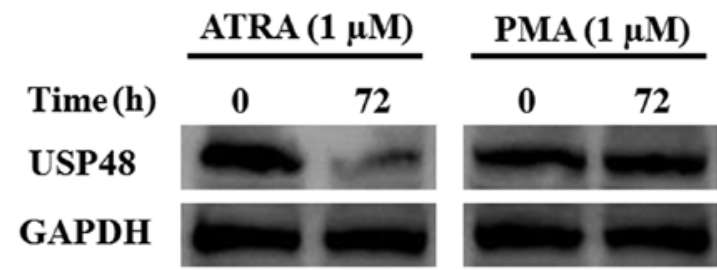

Figure 2. USP48 expression decreased during the differentiation of APL cells. USP48 expression at mRNA (above) and protein (below) levels were detected in (A) NB4 cells, and (B) HL60 cells following treatment with and without ATRA. (C) Western blotting was performed to detect USP48 expression in NB4 cells treated with ATRA and THP1 cells treated with PMA. ${ }^{* * *} \mathrm{P}<0.001$. USP48, ubiquitin-specific protease 48; APL, acute promyelocytic leukemia; ATRA, all-trans retinoic acid.

which indicated that NB4-cell proliferation was inhibited by overexpression of USP48 (Fig. 3A). The inhibition of cell proliferation by USP48 was further confirmed by cell cycle analysis (Fig. 3B). However, overexpression of USP48 did not affect the rate of apoptosis (Fig. 3C). To examine whether USP48 contributed to the ATRA-induced differentiation of APL cells, USP48 was overexpressed or silenced in NB4 cells. siUSP48-3 was the most effective oligonucleotide for silencing USP48 (Fig. 3D), and was selected for use in subsequent experiments. FCM analysis demonstrated that the expression of CD11b was increased following ATRA treatment of NB4 cells compared with control. Furthermore, CD11b expression was promoted by electroporation-mediated overexpression of USP48 in NB4 cells in response to ATRA treatment compared with untreated cells (Fig. 3E). In contrast, silencing of USP48 inhibited the expression of CD11b induced by ATRA in NB4 cells compared with untreated cells (Fig. 3E). The results demonstrated that USP48 inhibited the proliferation and promoted the ATRA-induced granulocytic differentiation of NB4 cells.

The function of USP48 is not dependent on the regulation of $p 65$.In further study of the underlying mechanisms of the role of USP48 in ATRA-induced granulocytic differentiation, western blotting demonstrated that the expression of USP48 increased in NB4 cells following ATRA treatment up to $24 \mathrm{~h}$, and decreased after $24 \mathrm{~h}$ (Fig. 4A). Furthermore, USP48 expression in the nucleus was decreased in the cytoplasm of NB4 cells treated with ATRA overall; however, this was preceded by an initial increase (Fig. 4B). Immunofluorescence staining also indicated that the localization of USP48 was predominantly in the nucleus of NB4 cells following ATRA treatment (Fig. 4C). These results implied that USP48 may function primarily in the nucleus during differentiation. The primary target of USP48 during ATRA-mediated differentiation was then investigated. The expression of p65, a reported target of USP48 in the nucleus (28), was suppressed by siRNA-mediated USP48 silencing in NB4 cells (Fig. 4D). This data indicated that the pathway mediated by nuclear factor $-\kappa \mathrm{B}(\mathrm{NF}-\kappa \mathrm{B})$ in ATRA-induced differentiation may contribute to the function of USP48. However, immunofluorescence staining revealed inconsistent findings, as p65 and USP48 were not co-localized in NB4 cells following ATRA treatment (Fig. 4E). These results suggested that the function of USP48 in ATRA-induced differentiation may not depend on the regulation of $\mathrm{p} 65$.

USP48 is regulated by miR-301a-3p. A total of 3 candidate microRNAs, miR-148a-3p, miR-301a-3p and miR-454a-3p, were selected based on chip results of microRNA expression profiles (data not shown). The expression of the candidate microRNAs in NB4 cells was confirmed by RT-qPCR: The expression of miR-148a-3p was decreased and that of miR-301a-3p was significantly increased by ATRA compared with untreated cells. However, no significant change in the 
A

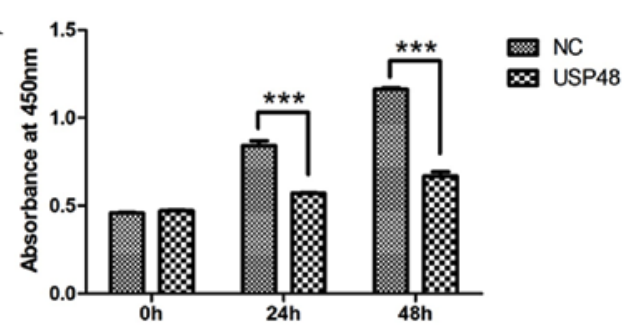

C

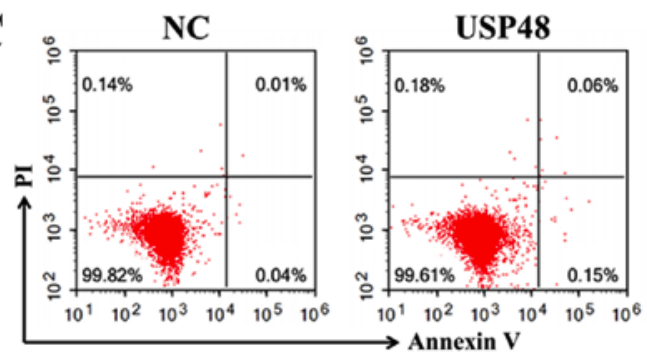

B

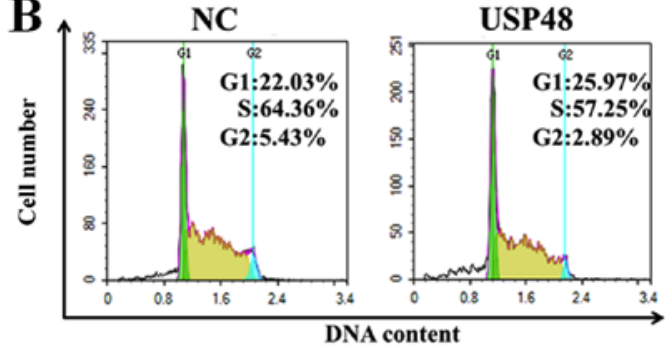

D
E

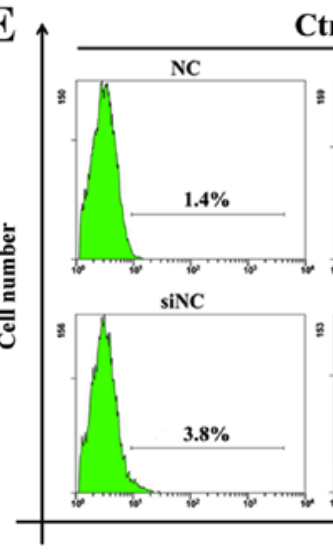

Ctrl

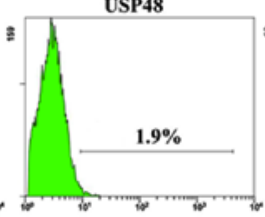

siUSP48

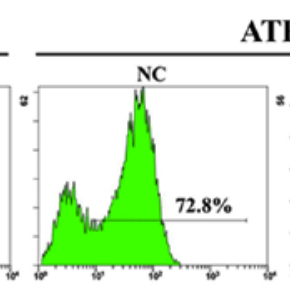

ATRA
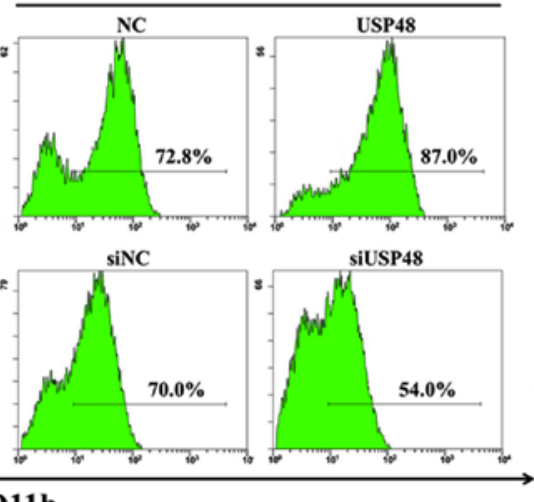
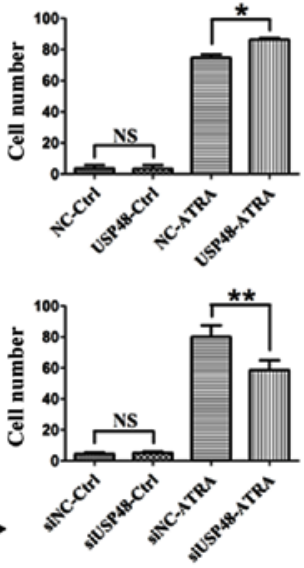

Figure 3. USP48 inhibits the proliferation and promotes ATRA-induced differentiation of NB4 cells. (A) Cell proliferation was measured by CCK-8 assay following overexpression of USP48 in NB4 cells. (B) Cell cycle analysis was performed by flow cytometry following overexpression of USP48 in NB4 cells. (C) Cell apoptosis was analyzed by Annexin V/PI staining following overexpression of USP48 in NB4 cells. (D) Silencing efficiency was validated by western blotting following knockdown of USP48 using siRNA. (E) The synthesis of CD11b was detected by flow cytometry in NB4 cells treated with or without ATRA following electroporation-mediated overexpression or knockdown of USP48. ${ }^{*} \mathrm{P}<0.05,{ }^{* *} \mathrm{P}<0.01$ and ${ }^{* * *} \mathrm{P}<0.001$. USP48, ubiquitin-specific protease 48 ; ATRA, all-trans retinoic acid; CCK-8, cell counting kit-8; PI, propidium iodide, si, small interfering RNA.

expression of miR-454a-3p was observed (Fig. 5A). Therefore, miR-301a-3p was selected for further evaluation of the regulation of USP48 expression. Western blot analysis demonstrated that the expression of USP48 was decreased by transfection with miR-301a-3p mimics, and increased by transfection with miR-301a-3p inhibitor (Fig. 5B). To determine whether USP48 was an miR-301a-3p target gene, the 3'UTR of the cDNA transcript was examined using TargetScanHuman 7.0. A 7mer-m8 binding site for miR-301a-3p was identified, located at position 174-180 of the 3'UTR (Fig. 5C). To determine whether USP48 was a direct target of miR-301a-3p, luciferase reporter vectors harboring USP48-WT and USP48-Mut were constructed. Co-transfection of miR-301a-3p mimics and the luciferase-USP48-WT fusion construct resulted in decreased luciferase activity compared with $\mathrm{NC}$ mimics-luciferase-USP48-WT fusion. However, this effect was not evident with transfection of the luciferase-USP48-Mut fusion construct (Fig. 5D). These observations suggested that miR-301a-3p may regulate USP48 via the binding site in the 3'UTR.
Upregulation of USP48 expression is a potential positive prognostic indicator in $A M L$. cBioPortal results indicated that USP48 was upregulated in the majority of the considered patients with AML (Fig. 6A). Furthermore, upregulation of USP48 in AML may be associated with increased overall survival time (median, 25.8 months in patients exhibiting overexpressed USP48 vs. 17.4 months in the remaining patients; log-rank test, $\mathrm{P}=0.356$; Fig. 6B). Furthermore, upregulation of USP48 expression may be associated with increased disease-free survival in AML (log-rank test, $\mathrm{P}=0.153$; Fig. $6 \mathrm{C}$ ). However, these associations were not statistically significant, which may be due to the small samples sizes. These analyses should be repeated using large cohorts, to confirm whether upregulation of USP48 expression is predictive of good prognosis in AML.

\section{Discussion}

Although the biological functions and substrates of the majority of DUBs remain unclear, DUBs have been suggested 


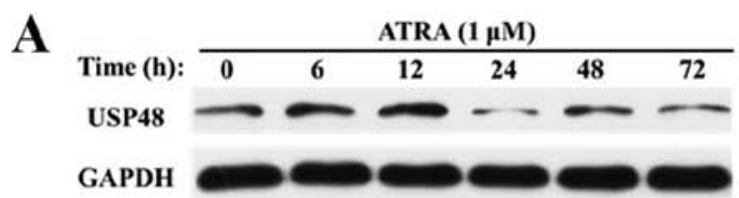

$\mathbf{B}$

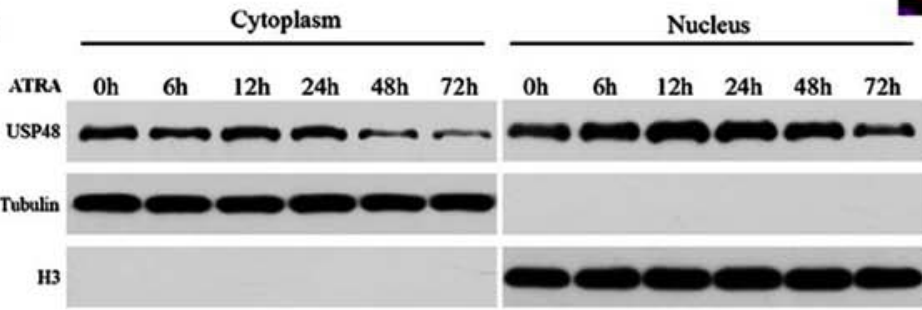

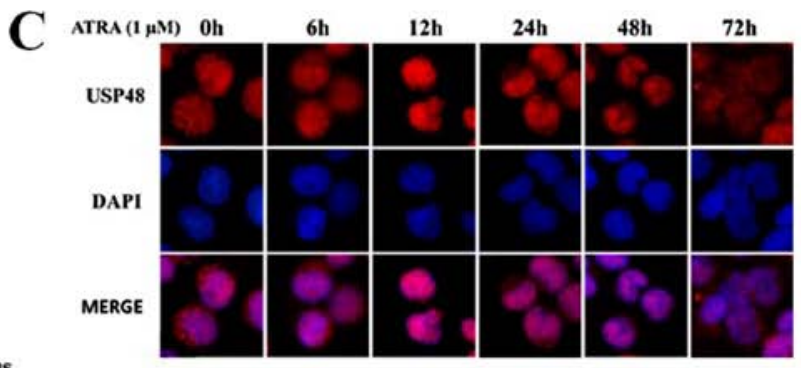

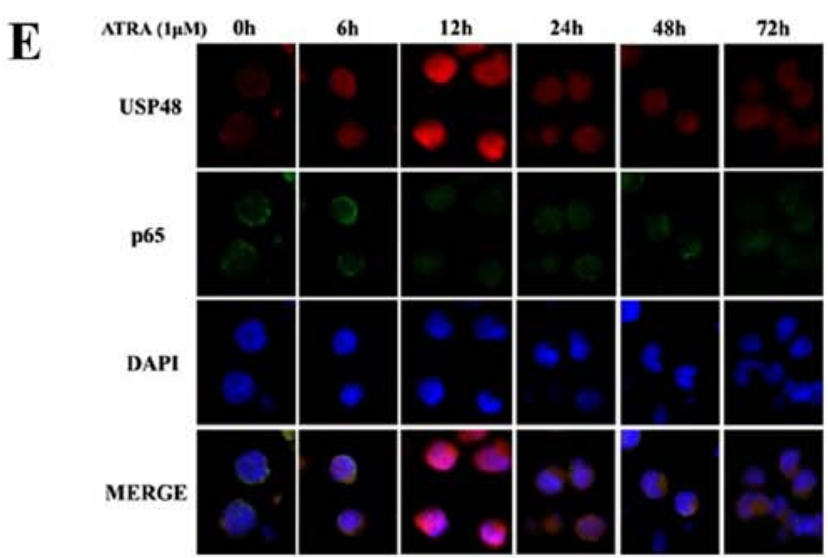

Figure 4. The function of USP48 does not depend on the regulation of p65. Western blotting was performed to detect USP48 protein expression in (A) total protein, and (B) cytoplasm and nucleus protein extracts of NB4 cells following treatment with ATRA. (C) The localization of USP48 in NB4 cells following ATRA treatment was detected by immunofluorescence staining. (D) p65 protein expression was detected by western blotting following USP48 overexpression in NB4 cells. (E) The co-localization of USP48 and p65 in NB4 cells following ATRA treatment was analyzed using immunofluorescence staining. USP48, ubiquitin-specific protease 48; ATRA, all-trans retinoic acid; si, small interfering RNA; NC, negative control.

A

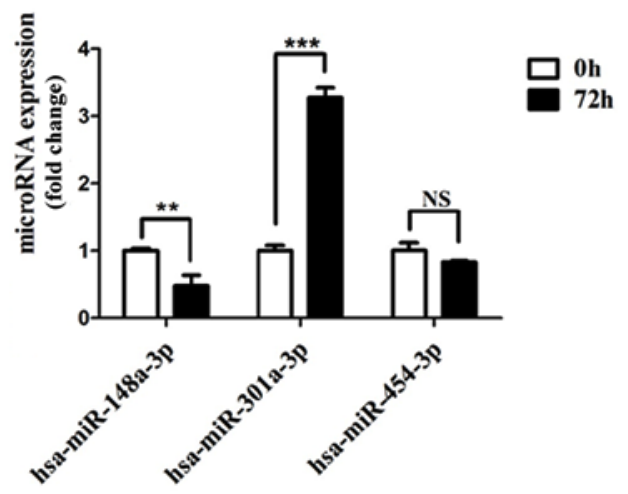

C

$$
\text { USP48 3' UtR-WT } 5^{\prime} \text {......GUCAaAuaAagcagauUgcacug...... }
$$$$
\text { USP48 3' UTR-Mut } 5^{\prime} \text { '......GUCAAAUAAAGCAGAAACGUGAG...... }
$$

B

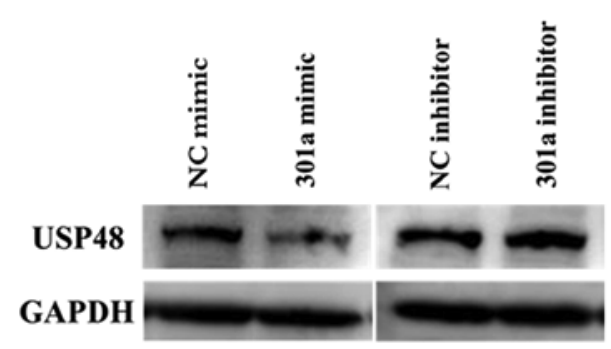

D

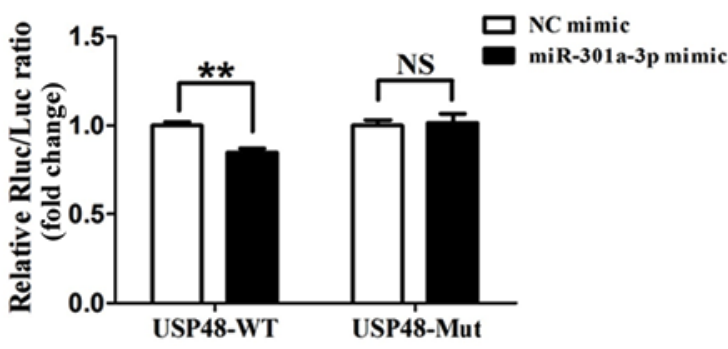

Figure 5. USP48 expression is regulated by miR-301a-3p. (A) The expression of 3 microRNAs in NB4 cells following treatment with ATRA. (B) The protein expression levels of USP48 following overexpression/knockdown of miR-301a-3p. (C) The binding site between USP48 and miR-301a-3p. (D) Luciferase activities following $3^{\prime} \mathrm{UTR}$ and miR-301a-3p transfection. ${ }^{* *} \mathrm{P}<0.01$ and ${ }^{* * *} \mathrm{P}<0.001$. USP48, ubiquitin-specific protease 48; miR, micro RNA; ATRA, all-trans retinoic acid; WT, wild type; Mut, mutated; NC, negative control; NS, not significant; Rluc, Renilla luciferase; Luc, Firefly luciferase. 

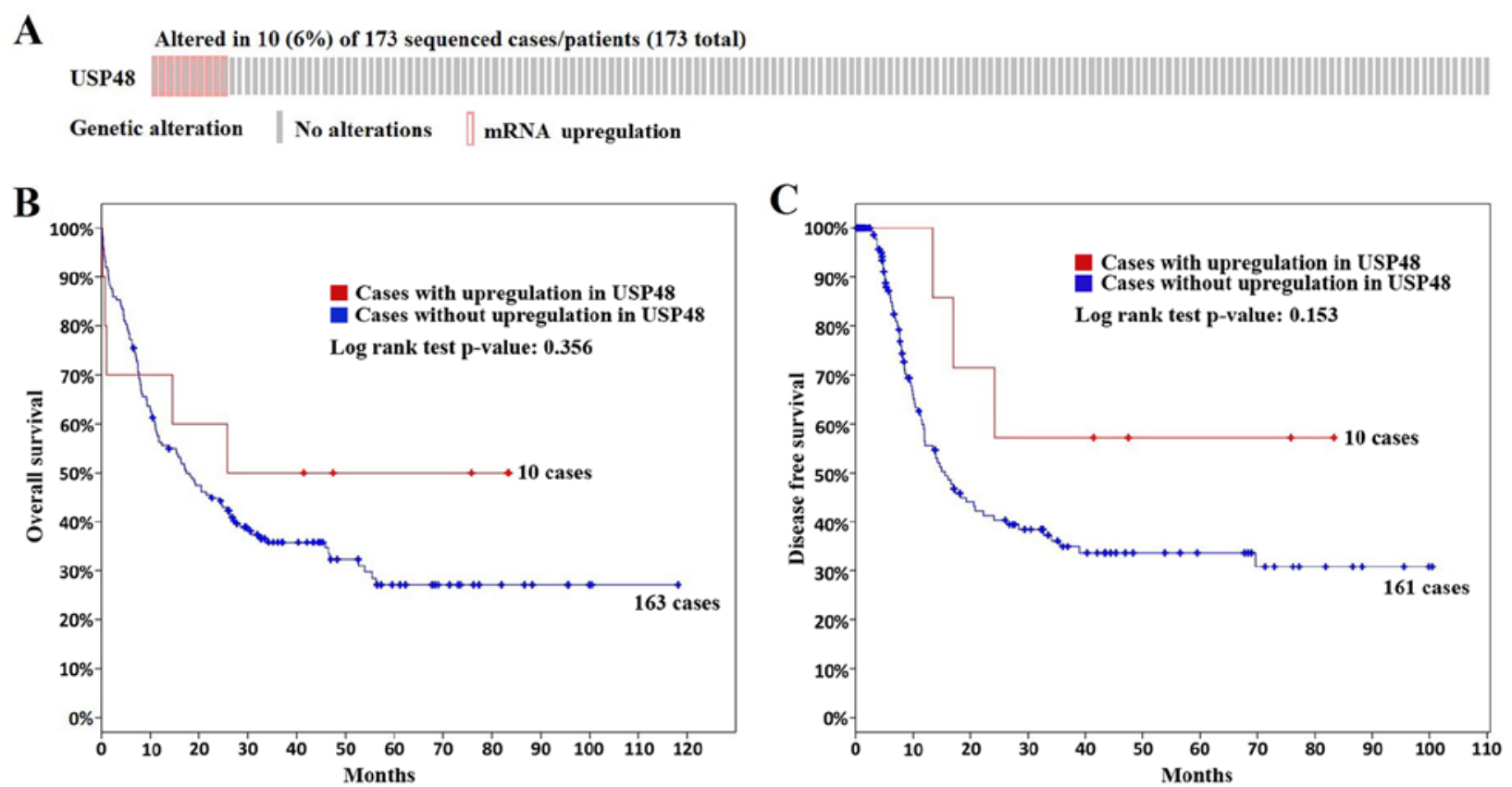

Figure 6. Upregulation of USP48 expression may be predictive of survival in AML according to the cBioPortal results. (A) In patients with AML, upregulation was the primary alteration of USP48 expression. (B) Overall survival and (C) disease-free survival analyses are presented as a Kaplan-Meier curves generated by cBioPortal. USP48, ubiquitin-specific protease 48; AML, acute myeloid leukemia.

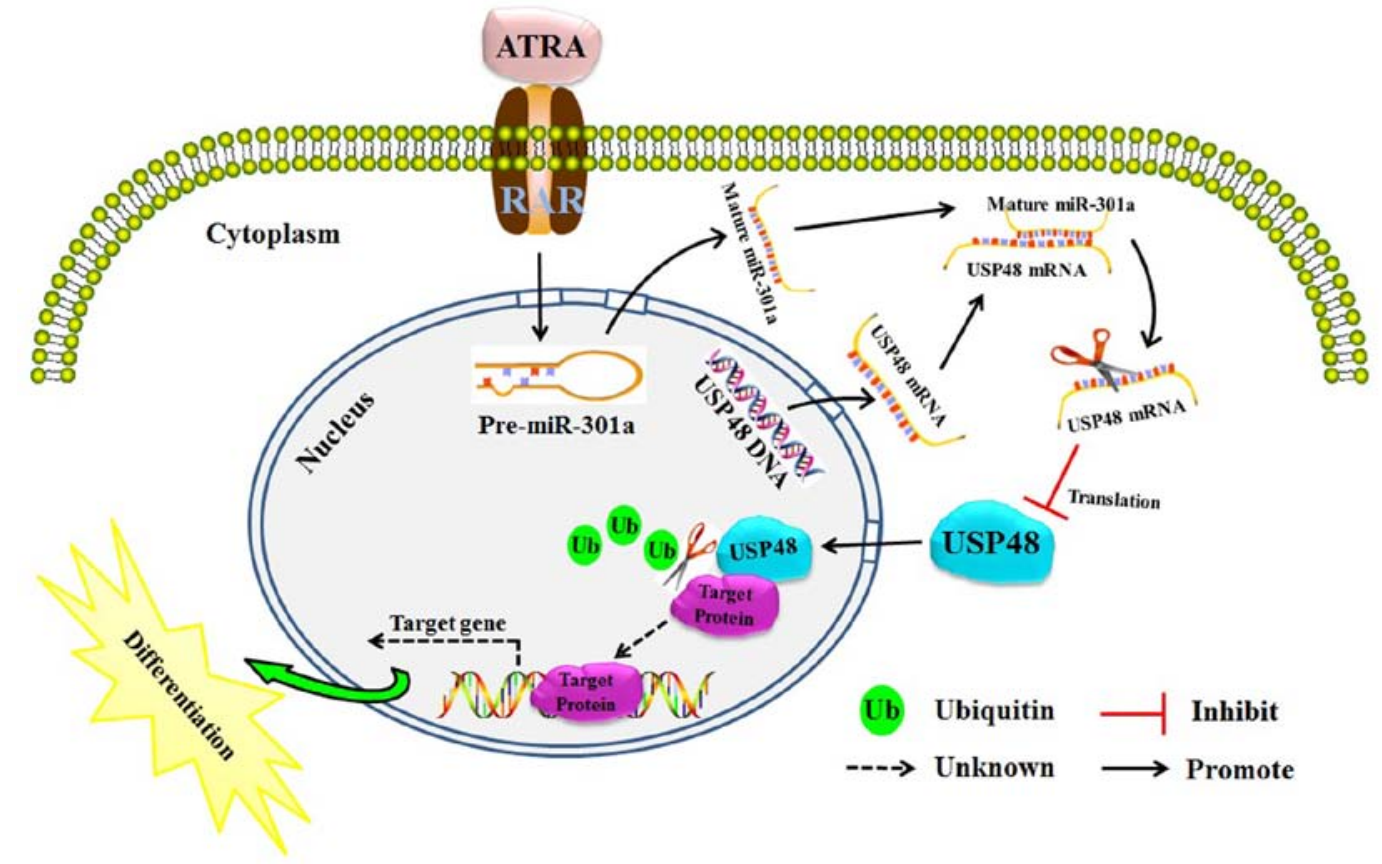

Figure 7. A putative model illustrates the role of USP48 in ATRA-induced differentiation of APL cells. USP48, ubiquitin-specific protease 48; ATRA, all-trans retinoic acid; APL, acute promyelocyticleukemia; RAR, retinoic acid receptor.

to be important targets for the treatment of human diseases, including cancer. For example, USP9X has been demonstrated regulate the destruction of Ets-1, which serves important functions in the tumorigenic program of metastatic melanoma (29). Additionally, USP15 has been demonstrated to bind to and stabilize p53, which serves a critical role in cancer progression through deubiquitination (30). A previous study reported that USP49 deubiquitinates and stabilizes FKBP51 to negatively regulate tumorigenesis and chemoresistance through AKT signaling (31). In addition, USP4-mediated deubiquitination and stabilization of PRL-3 is required for potentiating colorectal oncogenesis (32). In the present study, deubiquitinase USP48 was identified by microarray analysis, which was performed to comparatively evaluate the expression profile of HL60 cells following ATRA-induced differentiation. USP48 expression was inhibited significantly following ATRA exposure. Thus, the present study highlights USP48 as a potential therapeutic target for APL. 
Recently, it has been reported that USP48 can regulate the $\mathrm{NF}-\kappa \mathrm{B}$ signaling pathway by regulating the stability of the RelA (p65) in the nucleus (28). Furthermore, previous study indicated that USP48 regulates the stability of Mdm2 protein and thus the p53 signaling pathway, which is not dependent on its activity as a ubiquitination enzyme (33). In addition, USP48 has been demonstrated to reduce E-cadherin-mediated adherens junctions through increasing TNF receptor associated factor 2 stability (34). Furthermore, high expression of USP48 has been correlated with glioma malignancy, and USP48 has been demonstrated to activate Gli-dependent transcription and stabilize Gli1 protein through direct cleavage of its ubiquitin, which is critical for glioma cell proliferation and glioblastoma tumorigenesis (35). A previous study predicted that an imbalance in the BRCA1-BRCA1 associated RING domain 1-USP48 circuit has deleterious consequences for genome stability and that it may have significance in the prevention and progression of cancer (36). Therefore, numerous studies indicate that USP48 is involved in immunoregulation and cancer pathogenesis, which further prompted the clarification of its function in myeloid differentiation in the present study.

To investigate whether USP48 contributes to the degradation of the is promyelocytic leukemia-retinoic acid receptor $\alpha$ (PML-RAR $\alpha$ ) fusion protein in response to ATRA exposure, a molecular mechanism that can induce myeloid differentiation, NB4 (PML-RAR $\alpha$ positive) and HL60 (PML-RAR $\alpha$ negative) cells were used (37). The results revealed that USP48 expression was inhibited in NB4 and HL60 cells treated with ATRA compared with untreated cells. This data indicated that the expression of USP48 may not be dependent on the degradation of PML-RAR $\alpha$. To elucidate the mechanism of USP48 function in ATRA-mediated differentiation, the co-localization of p65 and USP48 after ATRA exposure using immunofluorescence staining. p65 protein expression was not co-localized with that of USP48, suggesting that USP48 may regulate ATRA-induced differentiation of APL cells via other signaling pathways. Furthermore, the possible regulation of USP48 by miR-301a-3p was investigated. Reduced USP48-promoter activity was observed upon miR-301a-3p treatment in the luciferase assay; however, the effect was not significant. Thus, USP48 may be partially regulated by miR-301a-3p. It is believed that USP48 expression is induced by the Sonic Hedgehog pathway through Gli1-mediated transcriptional activation (35) and that its ubiquitin chain trimming activity is regulated by casein-kinase-2-mediated phosphorylation in response to cytokine-stimulation (28). Therefore, USP48 may serve an important role in leukemia-cell differentiation, however, the underlying mechanism requires further investigation. In addition to USP48, other DUBs have been associated with leukemia. For example, HAUSP (USP7) aberrantly regulates the nuclear exclusion of the tumor suppressor phosphate and tensin homolog in APL via deubiquitinase activity (38). Other DUBs, including USP9X (39), CYLD (40) and A20 (41), are associated with the occurrence and ATRAinduced differentiation of leukemia cells. In consistence with previous studies, the present study suggests that DUBs function in ATRA-induced differentiation of leukemia cells.

In summary, it was demonstrated that USP48 inhibits the proliferation of leukemia cells and promotes ATRA-induced differentiation of leukemia cells. In addition, the expression of USP48 was demonstrated to be partially regulated by miR-301a-3p. Therefore, the present study eludes to a previously unknown miR-301a-3p-USP48 molecular network, which regulates the differentiation of leukemia cells (Fig. 7). This implies that dysregulation of USP48 may underlie the abnormal differentiation in APL, and that USP48 is a potential therapeutic target for APL.

\section{Acknowledgements}

Not applicable.

\section{Funding}

The present study was supported by the grants from the Shandong Medical and Health Science and Technology Plan Project (grant nos. 2015WS0193 and 2014WS0067), the Foundation for Outstanding Young Scientists in Shandong Province (grant no. 2014BSC03013) and the Innovation Project of the Shandong Academy of Medical Sciences.

\section{Availability of data and materials}

The analyzed data sets generated during the present study are available from the corresponding author on reasonable request.

\section{Authors' contributions}

LLL wrote the manuscript. LLL, HYL and GSJ designed the experiments, LLL, YW, XYZ, GHS, QG, ZYZ, YTD and HPY performed the experiments and analyzed the data. The final version of the manuscript has been read and approved by all authors.

\section{Ethics approval and consent to participate}

Not applicable.

\section{Consent for publication}

Not applicable.

\section{Competing interests}

The authors declare that they have no competing interests.

\section{References}

1. Fialkow PJ, Janssen JW and Bartram CR: Clonal remissions in acute nonlymphocytic leukemia: Evidence for a multistep pathogenesis of the malignancy. Blood 77: 1415-1417, 1991.

2. Mistry AR, Pedersen EW, Solomon E and Grimwade D: The molecular pathogenesis of acute promyelocytic leukaemia: Implications for the clinical management of the disease. Blood Rev 17: 71-97, 2003.

3. Nasr R, Guillemin MC, Ferhi O, Soilihi H, Peres L, Berthier C, Rousselot P, Robledo-Sarmiento M, Lallemand-Breitenbach V, Gourmel B, et al: Eradication of acute promyelocytic leukemiainitiating cells through PML-RARA degradation. Nat Med 14: 1333-1342, 2008.

4. Dos Santos GA, Kats L and Pandolfi PP: Synergy against PML-RARa: Targeting transcription, proteolysis, differentiation, and self-renewal in acute promyelocytic leukemia. J Exp Med 210: 2793-2802, 2013. 
5. Dombret H, Castaigne S, Fenaux P, Chomienne C and Degos L: Induction treatment of acute promyelocytic leukemia using all-trans retinoic acid. Controversies about dosage, advantages and side-effect management. Leukemia 8 (Suppl 3): S73-S75, 1994.

6. Tomita A, Kiyoi $\mathrm{H}$ and Naoe T: Mechanisms of action and resistance to all-trans retinoic acid (ATRA) and arsenic trioxide $\left(\mathrm{As}_{2} \mathrm{O}_{3}\right)$ in acute promyelocytic leukemia. Int J Hematol 97: 717-725, 2013.

7. Wilkinson KD: Ubiquitination and deubiquitination: Targeting of proteins for degradation by the proteasome. Semin Cell Dev Biol 11: 141-148, 2000.

8. Goldberg AL: Protein degradation and protection against misfolded or damaged proteins. Nature 426: 895-899, 2003.

9. Ciechanover A: Proteolysis: From the lysosome to ubiquitin and the proteasome. Nat Rev Mol Cell Biol 6: 79-87, 2005.

10. Ciechanover A: Intracellular protein degradation: From a vague idea thru the lysosome and the ubiquitin-proteasome system and onto human diseases and drug targeting. Cell Death Differ 12: $1178-1190,2005$

11. Ciechanover A and Schwartz AL: Ubiquitin-mediated degradation of cellular proteins in health and disease. Hepatology 35 3-6, 2002.

12. Pickart CM: Mechanisms underlying ubiquitination. Annu Rev Biochem 70: 503-533, 2001.

13. Schulman BA: Twists and turns in ubiquitin-like protein conjugation cascades. Protein Sci 20: 1941-1954, 2011.

14. Komander D, Clague MJ and Urbé S: Breaking the chains: Structure and function of the deubiquitinases. Nat Rev Mol Cell Biol 10: 550-563, 2009

15. Komander D: Mechanism, specificity and structure of the deubiquitinases. Subcell Biochem 54: 69-87, 2010.

16. Amerik AY and Hochstrasser M: Mechanism and function of deubiquitinating enzymes. Biochim Biophys Acta 1695: 189-207, 2004.

17. Eletr ZM and Wilkinson KD: Regulation of proteolysis by human deubiquitinating enzymes. Biochim Biophys Acta 1843: $114-128,2014$.

18. Wilkinson KD: DUBs at a glance. J Cell Sci 122: 2325-2329, 2009

19. Nijman SM, Luna-Vargas MP, Velds A, Brummelkamp TR Dirac AM, Sixma TK and Bernards R: A genomic and functional inventory of deubiquitinating enzymes. Cell 123: 773-786, 2005.

20. Love KR, Catic A, Schlieker C and Ploegh HL: Mechanisms, biology and inhibitors of deubiquitinating enzymes. Nat Chem Biol 3: 697-705, 2007.

21. Quesada V, Díaz-Perales A, Gutiérrez-Fernández A, Garabaya C, Cal S and López-Otín C: Cloning and enzymatic analysis of 22 novel human ubiquitin-specific proteases. Biochem Biophys Res Commun 314: 54-62, 2004

22. Sowa ME, Bennett EJ, Gygi SP and Harper JW: Defining the human deubiquitinating enzyme interaction landscape. Cell 138 389-403, 2009

23. Livak KJ and Schmittgen TD: Analysis of relative gene expression data using real-time quantitative PCR and the 2(-Delta Delta C(T)) method. Methods 25: 402-408, 2001

24. Shi M, Ren X, Wang X, Wang H, Liu G, Yuan X, Zheng S, Yu L, Pan S, Song G, et al: A novel combination of oridonin and valproic acid in enhancement of apoptosis induction of HL-60 leukemia cells. Int J Oncol 48: 734-746, 2016.

25. Cerami E, Gao J, Dogrusoz U, Gross BE, Sumer SO, Aksoy BA, Jacobsen A, Byrne CJ, Heuer ML, Larsson E, et al: The cBio cancer genomics portal: An open platform for exploring multidimensional cancer genomics data. Cancer Discov 2: 401-404, 2012.

26. Gao J, Aksoy BA, Dogrusoz U, Dresdner G, Gross B, Sumer SO, Sun Y, Jacobsen A, Sinha R, Larsson E, et al: Integrative analysis of complex cancer genomics and clinical profiles using the cBioPortal. Sci Signal 6: pl1, 2013.
27. Ley TJ, Miller C, Ding L, Raphael BJ, Mungall AJ, Robertson A, Hoadley K, Triche TJ Jr, Laird PW, Baty JD, et al; Cancer Genome Atlas Research Network: Genomic and epigenomic landscapes of adult de novo acute myeloid leukemia. N Engl J Med 368 2059-2074, 2013

28. Schweitzer K and Naumann M: CSN-associated USP48 confers stability to nuclear NF- $\kappa \mathrm{B} /$ RelA by trimming K48-linked Ub-chains. Biochim Biophys Acta 1853: 453-469, 2015.

29. Potu H, Peterson LF, Kandarpa M, Pal A, Sun H, Durham A, Harms PW, Hollenhorst PC, Eskiocak U, Talpaz M, et al: Usp9x regulates Ets-1 ubiquitination and stability to control NRAS expression and tumorigenicity in melanoma. Nat Commun 8: 14449, 2017.

30. Liu WT, Huang KY, Lu MC, Huang HL, Chen CY, Cheng YL, Yu HC, Liu SQ, Lai NS and Huang HB: TGF- $\beta$ upregulates the translation of USP15 via the PI3K/AKT pathway to promote $\mathrm{p} 53$ stability. Oncogene 36: 2715-2723, 2017.

31. Luo K, Li Y, Yin Y, Li L, Wu C, Chen Y, Nowsheen S, Hu Q, Zhang L, Lou Z, et al: USP49 negatively regulates tumorigenesis and chemoresistance through FKBP51-AKT signaling. EMBO J 36: 1434-1446, 2017.

32. Xing C, Lu XX, Guo PD, Shen T, Zhang S, He XS, Gan WJ, Li XM, Wang JR, Zhao YY, et al: Ubiquitin-specific protease 4-mediated deubiquitination and stabilization of PRL-3 is required for potentiating colorectal oncogenesis. Cancer Res 76: 83-95, 2016.

33. Cetkovská K, Šustová H and Uldrijan S: Ubiquitin-specific peptidase 48 regulates $\mathrm{Mdm} 2$ protein levels independent of its deubiquitinase activity. Sci Rep 7: 43180, 2017.

34. Li S, Wang D, Zhao J, Weathington NM, Shang D and Zhao Y: The deubiquitinating enzyme USP48 stabilizes TRAF2 and reduces E-cadherin-mediated adherens junctions. FASEB J 32: 230-242, 2018

35. Zhou A, Lin K, Zhang S, Ma L, Xue J, Morris SA, Aldape KD and Huang S: Gli1-induced deubiquitinase USP48 aids glioblastoma tumorigenesis by stabilizing Gli1. EMBO Rep 18: 1318-1330, 2017.

36. Uckelmann M, Densham RM, Baas R, Winterwerp HH, Fish A, Sixma TK and Morris JR: USP48 restrains resection by sitespecific cleavage of the BRCA1 ubiquitin mark from H2A. Nat Commun 9: 229, 2018.

37. Song G, Shi L, Guo Y, Yu L, Wang L, Zhang X, Li L, Han Y, Ren X, Guo Q, et al: A novel PAD4/SOX4/PU.1 signaling pathway is involved in the committed differentiation of acute promyelocytic leukemia cells into granulocytic cells. Oncotarget 7: 3144-3157, 2016.

38. Song MS, Salmena L, Carracedo A, Egia A, Lo-Coco F, Teruya-Feldstein $\mathrm{J}$ and Pandolfi PP: The deubiquitinylation and localization of PTEN are regulated by a HAUSP-PML network. Nature 455: 813-817, 2008.

39. Schwickart M, Huang X, Lill JR, Liu J, Ferrando R, French DM, Maecker H, O'Rourke K, Bazan F, Eastham-Anderson J, et al: Deubiquitinase USP9X stabilizes MCL1 and promotes tumour cell survival. Nature 463: 103-107, 2010.

40. Espinosa L, Cathelin S, D'Altri T, Trimarchi T, Statnikov A, Guiu J, Rodilla V, Inglés-Esteve J, Nomdedeu J, Bellosillo B, et al: The Notch/Hes1 pathway sustains NF- $\kappa \mathrm{B}$ activation through CYLD repression in T cell leukemia. Cancer Cell 18: 268-281, 2010.

41. Novak U, Rinaldi A, Kwee I, Nandula SV, Rancoita PM, Compagno M, Cerri M, Rossi D, Murty VV, Zucca E, et al: The NF-\{kappa\}B negative regulator TNFAIP3 (A20) is inactivated by somatic mutations and genomic deletions in marginal zone lymphomas. Blood 113: 4918-4921, 2009. 